\title{
An Advanced 3D Printed Design of the Hybrid Infusion-Multielectrode Recording System for Local Field Potential and Single Unit Acquisition and Intrabrain Drug Delivery in Freely Moving Mice
}

\author{
DOI: 10.17691/stm2016.8.4.17
}

Received February 14, 2016

O. Senkov, PhD, Head of in vivo Electrophysiology Department';

A. Mironov, PhD, Associate Professor, Department of Neurotechnology, Institute of Biology and Biomedicine ${ }^{2}$;

Senior Researcher, Experimental Modeling Unit, Central Research Laboratory; Associate Professor,

Department of Normal Physiology named after N.Y. Belenkov3;

A. Dityatev, PhD, Professor, Head of Molecular Neuroplasticity Research Group ${ }^{1}$; Professor, Medical Faculty ${ }^{4}$

${ }^{1}$ German Center for Neurodegenerative Diseases (DZNE), 44/64 Leipziger St., Magdeburg, 39120, Germany;

2Lobachevsky State University of Nizhni Novgorod, 23 Prospekt Gagarina, Nizhny Novgorod, 603950,

Russian Federation;

${ }^{3}$ Nizhny Novgorod State Medical Academy, 10/1 Minin and Pozharsky Square, Nizhny Novgorod, 603005,

Russian Federation;

${ }^{4}$ Medical Faculty, Otto-von-Guericke University, 44 Leipziger St., 39120, Magdeburg, Germany

In our recent methodological paper (Senkov et al., 2015, Front Neurosci), we have introduced a highly versatile reusable hybrid infusion-multielectrode recording (HIME) system, which can be utilized in freely moving mice performing cognitive tasks, while their brain activity is being recorded electrophysiologically in terms of local field potential and at single unit resolution, and can be combined with local intrahippocampal or intracortical drug delivery a few hours before behavior experiments. Here, we describe a new more advanced version of the HIME system, where some of its parts can be quickly fabricated using modern 3D printing technology. Our preliminary results show that usage of $3 \mathrm{D}$ printers for the construction of small plastic parts of the system facilitates and speeds up the whole fabrication process and improves precision and reliability of HIME implants. It also improves wearability, safety and welfare of mice carrying the system on their heads in the postoperative period and during long-term behavior tests.

Key words: hybrid infusion-recording system; multielectrode system; electroencephalography; local field potentials; hippocampus; drugs delivery.

Introduction. Recently, Frontiers in Neuroscience has published our methodological paper in the "Neural Technology" section [1]. There we described a design of a new hybrid infusion-multielectrode recording (HIME) system for local field potential and provided detailed information how to build it from scratch by using inexpensive commonly available small parts. In short, we have used for electrode construction: tungsten wires $(\varnothing 50 / 112 \mu \mathrm{m})$ insulated with teflon (originally from A-M Systems, USA, distributed in Europe by Science Products, Germany) bound together in a stainless steel tubing $(\varnothing 0.7,0.5$, or $0.4 \mathrm{~mm}$ ), depending on number of wires inside. Each of such electrodes contains from 1 to 5 single tungsten wires, for example, to record from different cortical and/or hippocampal layers, and can be implanted simultaneously up to 6 different brain structures as we show in the paper, but if required, the number of electrodes can be easily increased, if implantation of guide cannulas is omitted. Instead of tungsten wires, other insulated metal wires can be used, such as stainless steel, or even non-movable tetrodes made of $4 \times$ formvar coated nichrome wires twisted and

For contacts: Oleg Senkov, e-mail: oleg.senkov@dzne.de 
melted by heat together $(\varnothing 18 / 25 \mu \mathrm{m})$. For interfacing electrodes with a 16-32 channels preamplifier, we used soldering-free "pin" technology, when each electrode wire is inserted into its own hole on the printed circuit electrode interface board (EIB-16 or EIB-32 by Neuralynx, USA) and electrical connection is realized through mechanical pinning the electrode wires by small golden pins. Instead of Neuralynx ElBs with Omnetics connectors, one can easily adapt to our HIME system any other boards available from www.open-ephys.org, www.arnoaarts.wix. com, www.adinstruments.com, www.trianglebiosystems. com and others, or even design one's own customized EIB and order it from a local printed circuitry boards fabrication company. For protection of fragile electrodes, cannulas and headstages on the mouse head from dust and damage during mice housing in animal facilities and during behavior experiments, we proposed to use an easy-to-make protection hat, which additionally serves as a Faraday cage, to reduce electromagnetic/electrostatic artifacts on EEG/units recording in freely moving mice.
Previously, we suggested building it from $5 / 10 \mathrm{ml}$ plastic syringes properly cut and covered with a grounded cupper or aluminum adhesive tape around the hat to function as a Faraday cage (See Figure (i)). Such design is very practical and can be made in any lab within a few minutes. However, it has some disadvantages, namely: 1) it has two ear-like plastic parts from the syringe on both the hat and the acrylic-cemented pedestal, which might be less ergonomic and comfortable for mice; 2 ) through these ear-like plastic parts two screws are inserted to hold the hat on the implanted pedestal, insertion of these screws requires some force to be applied, which may damage or displace the whole implant, if done improperly; 3 ) the protective hat is yet bulky and not customizable for different implants. To overcome all these drawbacks of the previous design, we decided to try to print all plastic parts by using a $3 \mathrm{D}$ printer.

Methods. All treatments and behavioral procedures were conducted in accordance with ethical animal research standards defined by German law and approved

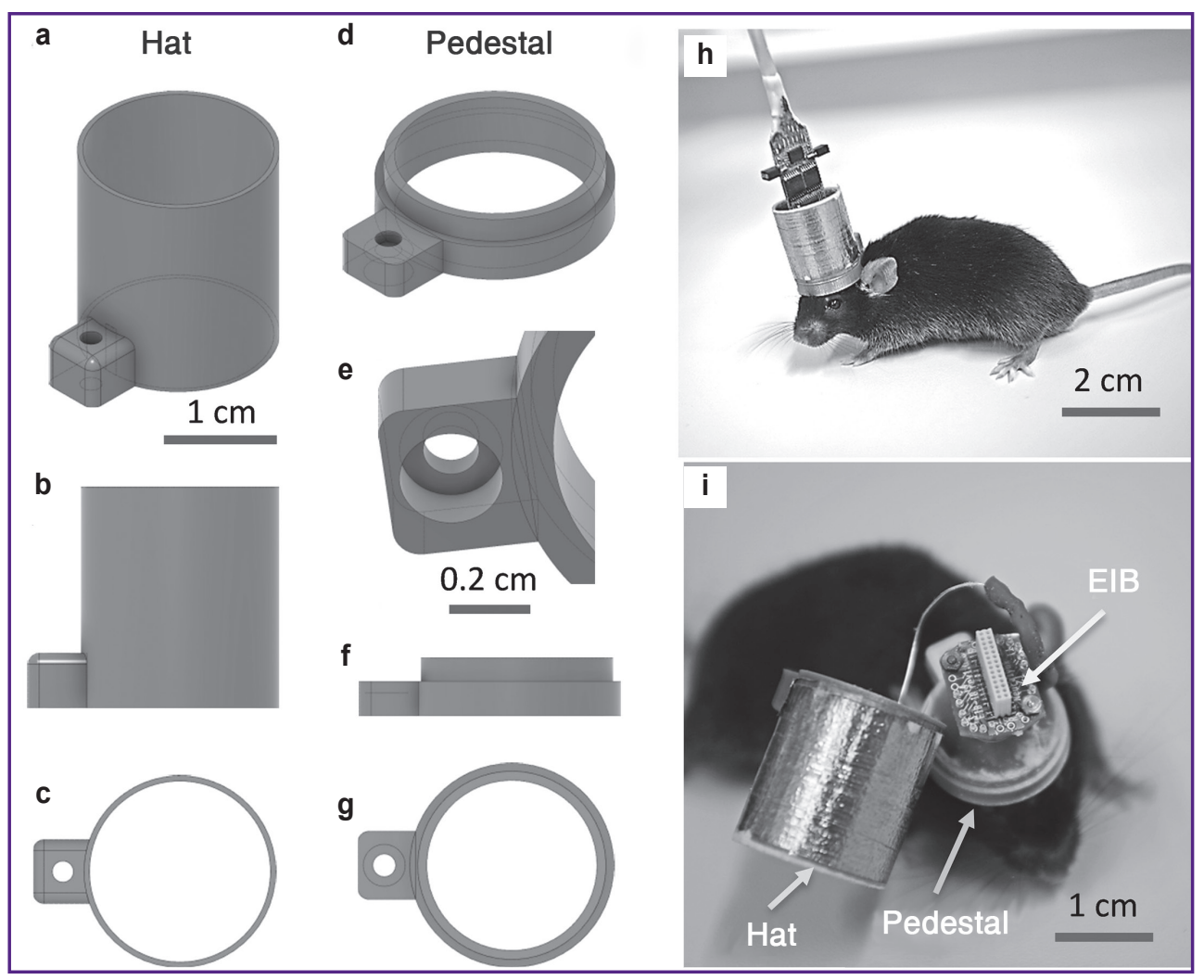

Design of 3D printed plastic parts for the HIME system: $(\mathrm{a})-(\mathrm{g}) 3 \mathrm{D}$ models made in Fusion 360 CAD software for printing with the 3D printer Ultimaker 2; (a)-(c) the protecting hat with a small hole $(\varnothing 2 \mathrm{~mm})$ for the $10 \mathrm{~mm} \mathrm{M1}$ screw to be used to hold the hat on the bottom pedestal; (d)-(g) the bottom pedestal which is supposed to be fixed with dental cement on the mouse head; (e) magnified view of the pedestal bottom part depicting a bigger hole $(\varnothing 3 \mathrm{~mm})$ for insertion a female screw for the M1 male screw, which is fixed there by melting plastic around with a hot soldering tip; (h) an example of implanted mouse bearing electrodes, 3D printed plastic parts and connected with a 16 channels Neuralynx preamplifier with a long tether/cable; (i) an awake mouse with the removed protecting hat exposing Neuralynx EIB-18, golden pins, electrodes, a ground wire and the dental acrylic fixed to the mouse cranium bone bottom pedestal 
by the Ethical Committee on Animal Health and Care of the State of Saxony-Anhalt, Germany; the license number: 42502-2-1159DZNE. Special care has been taken to minimize suffering, discomfort and number of animals used in the experiments.

To produce improved parts of the HIME system, we used the leading in EU Ultimaker 2 3D printer (www. ultimaker.com/en, Netherlands), which currently offers one of the best quality and precision of prints worldwide, up to $20 \mu \mathrm{m}$ layer resolution and speed of $24 \mathrm{~mm}^{3} / \mathrm{s}$. However, other modern 3D printers, e.g. with layer resolution 50 or $100 \mu \mathrm{m}$, can be used as well. For designing 3D models, we have utilized Fusion 360 3D CAD/CAM software www.autodesk.com, which is free for non-commercial application and academia. For slicing the 3D models before printing we used Cura, free software available at the Ultimaker website. In short (See Figure (a)-(g)), our EIB protecting hat 3D model has $18 \mathrm{~mm}$ in diameter, thickness of the walls just $1 \mathrm{~mm}$ and height of the hat $20 \mathrm{~mm}$. In overall, it takes about $20 \mathrm{~min}$ to print it with transparent PLA plastic filament (Verbatim, $2.85 \mathrm{~mm}$ thickness) with the following printing settings: layer height $100 \mu \mathrm{m}$, fill density $10 \%$, print speed $100 \mathrm{~mm} / \mathrm{s}$, nozzle size $0.4 \mathrm{~mm}$. After printing the weight of the hat was $\sim 1 \mathrm{~g}$, its surface was very smooth, and the whole construction was very robust, which allowed reusing the hat several times. The another part of the EIB hat is the implanted on the mouse head pedestal (See Figure (d)-(g)), which has the following parameters: outer diameter is also $18 \mathrm{~mm}$, inner diameter $15 \mathrm{~mm}$, height $5 \mathrm{~mm}$, printing time $10 \mathrm{~min}$, weight $0.5 \mathrm{~g}$. The whole implant including 3D printed parts, EIB, electrodes, pins, screws and Cupper adhesive tape around the hat weighs only $\sim 3 \mathrm{~g}$; together with dental cement it weighs 4-4.5 g (See Figure (h), (i)). Adult wildtype mice with weight $\geqslant 30 \mathrm{~g}$ can easy tolerate up to $6-7 \mathrm{~g}$ on their heads, due to very strong neck muscles, so the designed implant can be used in behavior experiments. Depending on the requirements, the protecting hat and the pedestal can be printed at least twice smaller, e.g. when only guide cannulas or an optical fiber for optogenetic stimulation are implanted, or less number of electrodes and thus smaller 8-channel-EIB is used.

Results. Testing the HIME system with 3D printed components, we found the following improvements, as compared with the previously published version: 1) it fits to mice more ergonomically and thus wearability is improved; 2 ) it is easier to restrain mice for $1-2 \mathrm{~min}$ by holding on the hat, e.g. while connecting them to the electrophysiological equipment; 3 ) it also became easier and risk-free for braking the implant to unscrew and remove the hat to expose electrodes/cannulas for manipulations, even on an awake mouse; 4) plastic parts can be printed in different colors, facilitating video tracking of mice in behavior experiments with special software, which can recognize multiple colors and efficiently trace them separately.

Thus, the advanced HIME system exemplifies how $3 \mathrm{D}$ printing technology brings more flexibility and potential in design and fabrication of state-of-art neuroscience tools, with a good "quality/costs" ratio and improved characteristics. The improved HIME system can be used for experiments involving injection of extracellular proteases, such as matrix metalloproteinase MMP-9 [2] or enzymes that digest particular glycans, such as endoneuraminidase (EndoNF) for polysialic acid [3], chondroitinase $A B C$ for chondroitin sulfates [4] and heparinase for heparan sulfates [5]. The functions of these and other carbohydrates carried by cell adhesion and extracellular matrix molecules have been mostly studied in vitro [6, 7], and we anticipate that our HIME system will facilitate translation of in vitro insights into mechanistic in vivo studies.

Acknowledgements. The research was supported by the Federal Target Program "Research and development in priority areas of the development of the scientific and technological complex of Russia for 2014-2020" of the Ministry of Education and Science of Russia, contract 14.581.21.0016 (Project ID RFMEFI58115X0016).

Conflicts of Interest. The authors declare no conflicts of interest.

\section{References}

1. Senkov O., Mironov A., Dityatev A. A novel versatile hybrid infusion-multielectrode recording (HIME) system for acute drug delivery and multisite acquisition of neuronal activity in freely moving mice. Front Neurosci 2015; 9: 425, https://doi. org/10.3389/fnins.2015.00425.

2. Wlodarczyk J., Mukhina I., Kaczmarek L., Dityatev A. Extracellular matrix molecules, their receptors, and secreted proteases in synaptic plasticity. Dev Neurobiol 2011; 71(11): 1040-1053, https://doi.org/10.1002/dneu.20958.

3. Kochlamazashvili G., Senkov O., Grebenyuk S., Robinson C., Xiao M.F., Stummeyer K., Gerardy-Schahn R., Engel A.K., Feig L., Semyanov A., Suppiramaniam V., Schachner M., Dityatev A. Neural cell adhesion moleculeassociated polysialic acid regulates synaptic plasticity and learning by restraining the signaling through GluN2Bcontaining NMDA receptors. J Neurosci 2010; 30(11): 4171 4183, https://doi.org/10.1523/JNEUROSCI.5806-09.2010.

4. Pizzorusso T., Medini P., Landi S., Baldini S., Berardi N. Maffei L. Structural and functional recovery from early monocular deprivation in adult rats. Proc Natl Acad Sci USA 2006, 103(22): 8517-8522, https://doi.org/10.1073/pnas.0602657103.

5. Korotchenko S., Cingolani L.A., Kuznetsova T., Bologna L.L., Chiappalone M., Dityatev A. Modulation of network activity and induction of homeostatic synaptic plasticity by enzymatic removal of heparan suffates. Philos trans $R$ Soc Lond B Biol Sci 2014, 369(1654): 20140134, https://doi. org/10.1098/rstb.2014.0134.

6. Dityatev A., Frischknecht R. Seidenbecher C.I. Extracellular matrix and synaptic functions, Results Probl Cell Differ 2006, 43: 69-97, https://doi, org/10, 1007/400_025.

7. Senkov O.. Andjus P. Radenovic L., Soriano E., Dityater A. Neural ECM molecules in synaptic plasticity, learning, and memory. Prog Brain Res 2014; 214: 53-80, https://doi.org/10.1016/B978-0-444-63486-3.00003-7. 